\title{
ER Stress and Iron Homeostasis: A New Frontier for the UPR
}

\author{
Susana J. Oliveira, ${ }^{1,2}$ Maria de Sousa, ${ }^{1,2}$ and Jorge P. Pinto ${ }^{1}$ \\ ${ }^{1}$ Instituto de Biologia Molecular e Celular, Universidade do Porto, Rua do Campo Alegre 823, 4150-180 Porto, Portugal \\ ${ }^{2}$ Instituto de Ciências Biomédicas Abel Salazar, Largo Prof. Abel Salazar 2, 4099-003 Porto, Portugal \\ Correspondence should be addressed to Maria de Sousa, mdesousa@ibmc.up.pt
}

Received 12 July 2010; Accepted 1 October 2010

Academic Editor: Emil Pai

Copyright ( $) 2011$ Susana J. Oliveira et al. This is an open access article distributed under the Creative Commons Attribution License, which permits unrestricted use, distribution, and reproduction in any medium, provided the original work is properly cited.

The C282Y mutation of HFE accounts for the majority of cases of the iron overload disease Hereditary Hemochromatosis (HH). The conformational changes introduced by this mutation impair the HFE association with $\beta_{2}$-microglobulin $\left(\beta_{2} \mathrm{~m}\right)$ and the cell surface expression of the protein: with two major consequences. From a functional perspective, the ability of HFE to bind to transferrin receptors 1 and 2 is lost in the C282Y mutant, thus affecting hepcidin regulation. Also due to the faulty assembly with $\beta_{2} \mathrm{~m}$, HFE-C282Y molecules remain in the endoplasmic reticulum (ER) as aggregates that undergo proteasomal degradation and activate an Unfolded Protein Response (UPR). UPR activation, regardless of the ER stress stimuli, was shown to reshape the expression profile of iron-related genes and to decrease MHC-I cell surface expression. The possibility of a HFE-C282Y-mediated interplay between the UPR and iron homeostasis influencing disease progression and the clinical heterogeneity among C282Y carriers is discussed. The responsiveness of the ER chaperone calreticulin to both ER and iron-induced oxidative stresses, and its correlation with HH patients' phenotype, reinforce the interest of dissecting the UPR signaling/iron metabolism crosstalk and points to the potential clinical value of use of pharmacological chaperones in HFE-HH.

\section{Introduction}

Occupying a central position in the secretory route, the endoplasmic reticulum (ER) performs a vast array of functions that includes the biosynthesis, folding, assembly, and posttranslational modification of secretory and membranetargeted proteins [1]. The accuracy of this variety of processes relies on specialized luminal conditions, thoroughly maintained by stringent quality control mechanisms [2]. Despite the sophistication of such mechanisms, certain physiological states and exogenous stimuli can compromise this optimal folding environment and are generally referred to as ER stress [3].

The realization that there is a functional intersection between ER stress and iron metabolism emerged first from studies of the genetic disorder of iron overload, Hereditary Hemochromatosis $(\mathrm{HH})$ type 1 . Arising from a defective regulation of iron absorption, $\mathrm{HH}$ is intrinsically related to the gene HFE [4]. The product of this gene shares structural homology to a major histocompatibility complex class I (MHC-I) protein, requiring association with $\beta_{2}$ microglobulin $\left(\beta_{2} \mathrm{~m}\right)$ for cell surface expression $[5,6]$. In contrast to conventional MHC-I molecules, HFE is unable to bind peptides and has not been implicated in antigen presentation functions [6]. Instead, the HFE- $\beta_{2}$ m heterodimer was shown to bind transferrin receptor (TfR)1, thus competing for its interaction with diferric transferrin $[7,8]$. The binding capacity of HFE was later extended to TfR2 [9]. C282Y and $\mathrm{H} 63 \mathrm{D}$ are the two point mutations of HFE commonly underlying $\mathrm{HH}$ [5]. The former, resulting from a G-A transition that replaces the amino acid cysteine by tyrosine at position 282, is carried by the majority of $\mathrm{HH}$ patients (>85\%), while the H63D, consisting in a C-G transition that determines a histidine to aspartate substitution at position 63, acquires clinical significance in $\mathrm{C} 282 \mathrm{Y} / \mathrm{H} 63 \mathrm{D}$ compound heterozygotes $[10,11]$.

The surprise caused by the discovery of a MHC-Ilike protein partaking in iron homeostasis was diluted by previous research, fertile in reports describing immunological abnormalities in $\mathrm{HH}$ patients. Accordingly, higher 
$\mathrm{CD}^{+} / \mathrm{CD}^{+}$ratios [12], later attributed to defective numbers of $\mathrm{CD}^{+} \mathrm{T}$ cells [13], were consistently found in $\mathrm{HH}$ subjects in comparison to control individuals. Complementing these data, increased clinical severity of the disease was observed in the context of a lower $\mathrm{CD}^{+} \mathrm{T}$ lymphocyte pool [14]. Discrepancies at the functional level were likewise revealed, with $\mathrm{CD}^{+} \mathrm{T}$ cells from $\mathrm{HH}$ patients exhibiting impaired cytotoxic and CD8-p56lck activities when compared to healthy controls $[15,16]$. A glimpse for the structural homology with MHC-I molecules was also provided by the spontaneous iron overload phenotype developed in $\beta_{2} \mathrm{~m}^{-/-}$ mice [17].

The elucidation of the genetic background of $\mathrm{HH}$, in concert with the rapid adoption of HFE mutation testing in clinical practice, soon revealed a poor penetrance of the disease in C282Y carriers [18]. A remarkable phenotypic heterogeneity among patients was noticed as well [19], suggesting the existence of additional factors modifying this genetically determined disorder. Modifiers of the clinical and immunological traits of $\mathrm{HH}$ have been actively pursued, namely, genetic ones [20]. In light of the recent findings, the Unfolded Protein Response (UPR) has emerged as a promising candidate.

\section{The C282Y Mutation and the UPR}

By blocking the formation of a disulphide bond in the $\alpha_{3}$ domain of HFE, the C282Y mutation prevents the assembly with $\beta_{2} \mathrm{~m}[10,21]$. As a consequence, the mutant protein fails to progress through the secretory pathway and remains in the ER as high molecular weight aggregates $[10,22,23]$ that undergo proteasome-dependent degradation [10]. Besides the presence of such intracellular aggregates, cells expressing the C282Y mutant version of HFE also display decreased surface expression of MHC-I molecules [24]. Seeking the molecular details underlying this observation and inspired by the ER retention of the C282Y mutant, de Almeida and coworkers established the UPR as a mechanism mediating the HFE/MHC-I crosstalk [25]. Both the UPR/MHC-I interplay and the ability of the C282Y faulty HFE to elicit UPR activation were later independently confirmed [26, 27].

UPR is the designation of the specialized signaling circuits developed by cells to counteract the luminal accumulation of misfolded client proteins and rebalance the load/capacity ratio of the ER threatened by physiological states and exogenous conditions as diverse as potent secretory activity, disruption of $\mathrm{Ca}^{2+}$ stores, alteration of redox status, energy/nutrient deprivation, expression of mutant substrates, and viral infection $[28,29]$. Tailored to restore ER homeostasis, the UPR combines multiple synergistic strategies that encompass global suppression of protein synthesis and translocation into the ER, transcriptional induction of ER chaperones and foldases to face the increased folding demands, and improvement of the ER-associated degradation (ERAD) machinery to bolster the clearance of irreparably unfolded proteins $[3,30]$. If the prosurvival attempts are exhausted and ER damage prevails, UPRinduced proapoptotic programs are executed [31].
In mammalian cells, three ER-resident transmembrane proteins operate as proximal sensors and define the major UPR signaling pathways: double-stranded RNA-dependent protein kinase-like ER kinase (PERK), inositol-requiring enzyme (IRE) 1 , and activating transcription factor (ATF)6 $[3,30]$. Notwithstanding this diversity, association with the ER-resident chaperone immunoglobulin heavy chainbinding protein $(\mathrm{BiP})$ is proposed as a common regulator of the ER transducers. Under unstressed conditions, BiP binds the luminal domains of all sensors, rendering them inactive. As unfolded proteins congest the $\mathrm{ER}, \mathrm{BiP}$ is competitively titrated away, allowing the PERK-, IRE1-, and ATF6dependent cascades to proceed $[32,33]$. Alternative models of sensing have been presented as well. It was suggested, for example, that the MHC-like groove displayed by yeast IRE1 directly detects and binds misfolded clients in the ER lumen, which would contribute to IRE1 activation [34]. The groove dimensions in the related human protein, too narrow to accommodate peptides, have weakened the likelihood of this mechanism, however [35]. The two perspectives were recently reconciled by a model postulating that direct interaction with unfolded proteins is required for IRE1 activation, whereas BiP serves both as buffer and timer of UPR activity [36].

Encouraged by the misfolding nature of the C282Y HFE protein and the subsequent UPR activation, categorization of $\mathrm{HH}$ as a conformational disorder has been claimed [37, 38]. A reasonable explanation for the aforementioned genotypephenotype inconsistencies amongst $\mathrm{HH}$ patients could therefore rely, at least in part, on dissimilar individual abilities to mount an appropriate protective response towards the $\mathrm{C} 282 \mathrm{Y}$ mutant client. Albeit attractive, this hypothesis is far from consensual. One argument militating against it relates with the low tissue levels of HFE expression, recently estimated below $0.53 \mathrm{nmol} / \mathrm{g}$ of total protein in human liver [39]. Nonetheless, and despite some controversial data [40], increased hepatic mRNA expression of HFE was reported in iron-supplemented mice $[41,42]$, a trend also recapitulated by microglia-derived cells subjected to stressor agents and serum deprivation [43]. Accordingly, one could envisage a scenario in which the basally innocuous HFE pool may accumulate to levels that clog the ER with the C282Y misfolded variant as iron overload progresses in $\mathrm{HH}$, thus favoring ER stress conditions. Whether and how this vicious cycle-based model influences the in vivo pathophysiology of HFE-linked HH definitely deserves assessment.

\section{The UPR Crosstalk with Iron Metabolism}

3.1. Expression Profile of Iron-Related Genes. Originally described as a check-and-balance program focused on the recovery of stress-corrupted ER folding homeostasis, the boundaries of UPR have been steadily broadened to cell differentiation, metabolic and inflammatory processes [44-46]. Suggestive evidence for an intersection with iron metabolism was first provided fortuitously by differential gene expression screenings. Two such examples are the increased transcript levels of the ER chaperones calreticulin (CRT) and BiP found 
in iron-burdened astrocytoma cells [47] and the transferrin gene downmodulation reported in stable transfectants of the stress-inducible transcription factor CCAAT/enhancerbinding protein (C/EBP) homologous protein (CHOP) [48]. Likewise, proteomic analysis revealed increased hepatic BiP expression in dietary iron-loaded mice [49], although in vitro studies using the human hepatoma HepG2 cell line have failed to confirm this result (Pinto et al., unpublished data). Despite all these cues, the interplay of UPR and iron homeostasis has remained a hitherto unexplored field.

By exposing a hepatocyte-derived cell line to chemical agents impairing disulphide bond formation (dithiothreitol and homocysteine) in ER client proteins, we recently demonstrated that ongoing ER stress significantly reshapes the expression profile of iron-related genes, namely, hepcidin, ferroportin, and ferritin H. Using this experimental model, the molecular mechanism behind the biphasic modulation of hepcidin was also deciphered, with the nuclear factors $\mathrm{C} / \mathrm{EBP} \alpha$ and $\mathrm{CHOP}$ being implicated [50]. The interplay between iron metabolism and the UPR signaling pathways was independently corroborated by Vecchi et al. that, after stressing hepatoma-derived cells with the ER-to-Golgi transport inhibitor brefeldin A, calcium ionophore A23187 and tunicamycin, reported increased levels of hepcidin transcripts, a pattern also detected in the liver of tunicamycintreated mice. The stimulation of hepcidin was attributed to cAMP response element-binding protein $\mathrm{H}(\mathrm{CREBH})$ dependent activation of its promoter [51]. The mechanisms proposed by the two studies $[50,51]$ for hepcidin induction under stress scenarios are not incompatible and could conceivably coexist.

\subsection{Calreticulin, a Chaperone Related to Clinical Expression} of Iron Overload. As discussed earlier, UPR activation is characterized by the induction of several ER stress-responsive proteins. One of these molecules is CRT, a $46 \mathrm{kDa}$ ERresident lectin chaperone with $\mathrm{Ca}^{2+}$-buffering capacity, specifically devoted to the folding of a large fraction of clients traversing the secretory route, glycoproteins.

In addition to the chaperone function, CRT is involved in protection from stress usually not included in the UPRrelated ER stress category. CRT synthesis is induced by heat shock [52-54] and heavy metals [53], which has recently drawn attention to the role of this protein in protection from oxidative stress [55]. CRT, together with the other ER chaperone $\mathrm{BiP}$, protected renal epithelial cells against lipid peroxidation induced by the prooxidant reagent tert-butyl hydroperoxide [56]. Production of the antioxidant nitric oxide leads to overexpression of CRT in pancreatic beta cells [57]. Also, hypoxic conditions, such as those found in wounds, can lead to overexpression of CRT by 2 to 3 folds, with a 7-fold increase observed in response to $\mathrm{H}_{2} \mathrm{O}_{2}$-induced oxidative stress during rat cardiomyocyte injury [58].

A possible protective role for CRT was found in $\mathrm{C} 282 \mathrm{Y}$ homozygous $\mathrm{HH}$ patients, in which the iron-induced oxidative stress and an UPR triggered by the unfolded C282Y-mutated HFE protein coexists. In this study [59], a negative association was found between the levels of CRT

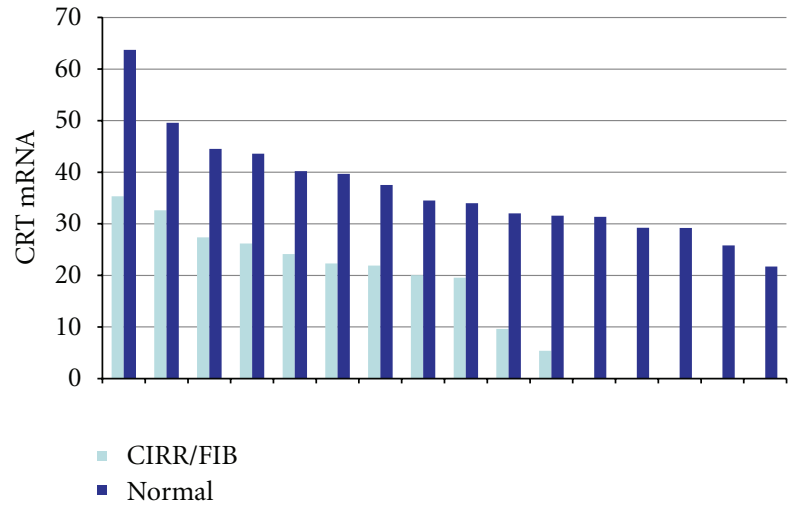

FIGURE 1: CRT expression and HH-related symptoms. CRT mRNA expression in PBMCs from HFE C282Y homozygous patients. Each bar represents an individual subject, ordered by CRT level and divided according to the presence (light blue bars) or absence (dark blue bars) of cirrhosis/fibrosis (CIRR/FIB), modified by Porto from original data from Pinto et al., 2008 [59].

in peripheral blood mononuclear cells (PBMCs) and the severity of $\mathrm{HH}$ clinical manifestations (Figure 1). Although the mechanism underlying the variability in CRT expression among $\mathrm{HH}$ patients has not been elucidated, the finding of a positive association between CRT mRNA levels, BiP expression, and numbers of monocytes (the PBMC population with the highest HFE expression) favors the interpretation that CRT expression is being modulated by the UPR in PBMCs from $\mathrm{C} 282 \mathrm{Y}^{+/+}$.

The involvement of CRT in cellular protection from oxidative stress is possibly the mechanism underlying the changes in CRT expression in response to increased intracellular iron levels observed in colorectal adenocarcinoma (Caco-2) and hepatocarcinoma (HepG2) cell lines [59, 60]. In the Núñez et al. study [60], the iron-induced increase in CRT expression was effectively blocked by the antioxidant quercetin, whereas Pinto et al. showed, using both over-expression and siRNA-mediated silencing, that CRT upregulation is necessary to prevent iron-induced ROS accumulation [59].

How could CRT exert its role in the protection of the cell against iron-induced oxidative stress? An answer might be given by mobilferrin, a cytosolic protein involved in the intracellular transport of iron during intestinal iron absorption [61]. Rat mobilferrin and CRT share 100\% homology in the amino-terminal amino acid sequence; both proteins have the same apparent molecular weight and isoelectric point, and the antibodies raised against one of the proteins cross-react with the other [62]. Mobilferrin is thus considered to be an isoform of CRT, although further studies are needed to confirm this hypothesis. The increase in CRT/mobilferrin in a situation of augmented intracellular iron levels (Figure 2) could be a response of the cell to the need for more iron-binding capacity, to ameliorate the iron-induced oxidative stress. In view of the presence of CRT in several intracellular (and extracellular) locations, this role could take place in a variety of compartments, 


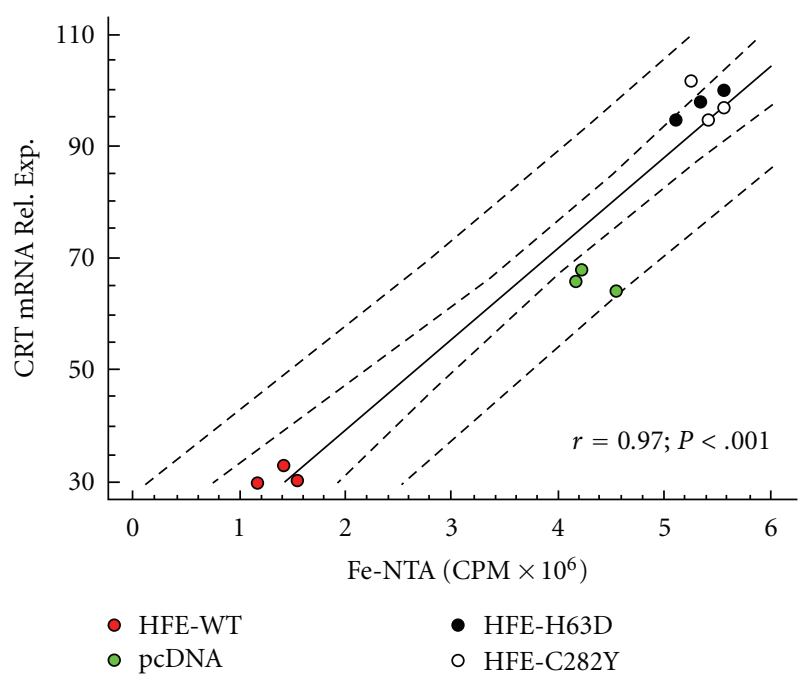

FIgURE 2: Regulation of CRT mRNA by iron. Linear regression analysis of the correlation between intracellular iron levels (3 $\mu \mathrm{M}$ ferric nitrilotriacetate ( $\left.{ }^{55} \mathrm{Fe}-\mathrm{NTA}\right)$ were used as iron source), and CRT mRNA pools in HepG2 cells overexpressing the WT or mutated (C282Y or H63D) HFE variants (HepG2 cells transfected with the empty pcDNA3 vector were used as negative control). Each dot represents the average of one experiment with three replicates. $r=0.97 ; P<.001$. Inner dashed curves represent $95 \%$ confidence intervals for the mean value of CRT at any selected CPM value. Outer dashed lines are $95 \%$ prediction limits for new observations. Modified from Pinto et al., 2008 [59].

where the need for iron buffering would be present. One such compartment is the nucleus, where the presence of hydroxyl radicals generates a whole series of DNA damage, namely, single- or double-strand breaks, abasic sites and base and sugar lesions $[63,64]$. Interestingly, over the last two decades, several studies have reported the presence of ferritin, the main iron-storage protein, in cell nuclei [65]. The earliest observations of nuclear ferritin were made in mice hepatocytes following iron overload [66]. Later, the presence of ferritin in the nucleus was found to correlate with higher resistance to UV and $\mathrm{H}_{2} \mathrm{O}_{2}$-induced DNA damage on corneal epithelial cells $[67,68]$, suggesting the need for protection from iron-induced oxidative stress in the nucleus.

CRT participation in the response to oxidative stress may be a component of the broader involvement of the ER in cellular protection from a varied category of insults, namely those involving the iron overload toxicity. The predominant location of CRT and other ER proteins within the hyperoxic environment of the ER increases their susceptibility to oxidative damage [69], which, along with posttranslational modifications, can affect both the function and cellular location of these proteins [70]. All concurrent hypotheses agree that oxidative stress-induced ER stress leads to extracellular release of CRT and other ER proteins. The mechanisms involved in the translocation of the protein to the extracellular compartment are not yet fully understood, although they seem to involve triggering of apoptotic cell regulatory proteins (caspases, Bap31, Bax activation), an ER stress response leading to the phosphorylation of the eukaryotic initiation factor eIF2 $\alpha$ and active exocytosis [71$74]$.

3.3. Calreticulin, at the Crossroads of MHC-I Expression and Iron Overload. Impaired cell surface expression of MHC-I molecules is another feature found in $\mathrm{C} 282 \mathrm{Y}^{+/+}$ hemochromatosis patients besides iron overload. In view of the UPR/MHC-I crosstalk revealed in recent years $[25,26]$, together with the iron burden developed by $\beta_{2} \mathrm{~m}$ and MHC-I KO murine models $[17,75]$, the chaperone activity of CRT might gain a renewed relevance in the context of MHCI assembly. The process takes place in the ER with the assistance of a number of chaperones and folding factors, which include CRT. The complex to be assembled consists of a glycosylated heavy chain, a $\beta_{2} \mathrm{~m}$ molecule, and a peptide. Peptide loading of MHC-I molecules is the final step of an intricate pathway that results from the adaptation of a quality control cycle that regulates the folding of conventional glycoproteins [76]. The complex responsible for peptide loading comprises the peptide transporter TAP (transporter associated with antigen processing), ERp57, CRT, calnexin (CANX), and tapasin (TAPBP, TAP-binding protein) [77, 78]. Both CRT and CANX promote the assembly of MHC-I and retain incompletely assembled complexes in the ER [78]. CRT binds to a monoglucosylated N-linked glycan at Asn86 of the MHC-I heavy chain with a dissociation constant of approximately $1 \mu \mathrm{M}$ [79], following CANX release.

The importance of CRT in MHC-I processing is illustrated by the suboptimal MHC-I assembly in CRT KO cells [80], with MHC-I expression and stability at the cell surface being rescued by the reintroduction of a lectin-deficient CRT mutant [81]. Although the mechanisms underlying the involvement of the MHC-I in iron metabolism remain unclear, the CRT role in MHC-I assembly/expression may, at least in part, explain the protective function of this protein in iron overload [59].

\section{The UPR Crosstalk with Iron Metabolism: Putative Physiological Significance}

The central regulator of iron homeostasis is hepcidin, a 25residue peptide hormone. Mainly secreted by hepatocytes [82], hepcidin binds to the membrane iron exporter ferroportin, triggering its internalization and lysosomal degradation [83]. Iron egress from enterocytes and macrophages is therefore inhibited, ultimately restricting the availability of the biometal in circulation. The pleiotropic nature of hepcidin arises from its responsiveness to iron, inflammation, anemia, and hypoxia $[84,85]$. Hepcidin is physiologically stimulated by increased iron stores and inflammation, with the converse occurring in the remaining conditions. This versatility is mirrored by the array of signaling pathways coordinating hepcidin transcription as yet identified [8691], now extended to the ER stress chain of events.

A systemic impact of the UPR has been disclosed through the connection to insulin secretion and peripheral resistance [92, 93], glucose homeostasis [94], and inflammation [95]. With the UPR-induced hepcidin modulation [50, 51], a new 
item can be coupled to this picture. By limiting duodenal iron absorption, hepcidin upregulation in this context may be part of a protective "strategy" to evade extra sources of stress, as those associated with iron-generated ROS. In line with hepcidin's antimicrobial role [82], consequences on the innate immunity are expected as well, thereby furthering the scope of the recently uncovered ER stress-mediated inflammatory responses $[46,96]$.

Besides the systemic impact underlined by hepcidin, repercussions of UPR activation are also manifested at the cellular iron metabolism level, as suggested by the modulation of ferroportin and ferritin $\mathrm{H}$ expression imposed by ER insults [50]. The mRNA enrichment of both genes in cells enduring ER stress may reflect an attempt to circumvent intracellular deposition of free iron either via its sequestration or export, respectively.

\section{The UPR Crosstalk with Iron Metabolism: A Link to Pathological Conditions}

Building evidence has coupled the ER stress response circuitries to multiple pathologies, namely diabetes, obesity, and neurodegeneration [97-99]. Apart from the abovedescribed connection with HFE-linked $\mathrm{HH}$, the novel association between ER stress and iron homeostasis may prove useful in furthering the understanding of neurodegenerative processes. In fact, iron accumulation in affected brain regions is a commonality of various neuropathologies, including Alzheimer's disease (AD) and Parkinson's disease (PD) [100]. Regardless of the yet uncertain mechanisms driving such deposition, the significance of inherent oxidative stress to neuronal damage has been increasingly recognized [101]. Belonging to the class of conformational disorders, protein misfolding and aggregation are also hallmarks of both $\mathrm{AD}$ and PD, probably potentiating neuronal cell death [99]. The neurodegeneration field may be therefore worth exploring for the dialogue between iron homeostasis and ER stress. One can conceive, for example, that the transcriptional reshaping triggered by UPR activation takes part on the brain iron imbalance observed in $\mathrm{AD}$ and $\mathrm{PD}$.

Another foreseeable repercussion of these new findings touches on the virus-iron metabolism-UPR defined triad. The ability of viruses to coopt the biochemical machineries of host cells to mass replicate themselves is a longstanding concept. One of the widely studied processes is the viral interference with multiple steps of MHC-class I antigen presentation route, likely evolved to elude immune surveillance [102]. Because iron availability is critical for efficient proliferation, an additional subversive approach triggered by viruses includes manipulation of host iron status. Despite our still tangential understanding of this strategy, progress has been made by demonstrating that TfR 1 might be engaged in the viral entry process [103, 104]. Furthermore, US2 and Nef proteins encoded in the genomes of human cytomegalovirus (HCMV) and human immunodeficiency virus (HIV)-1, respectively, were shown to down regulate the cell surface expression of HFE [105, 106], presumably with the consequence of replenishing intracellular iron stores and benefit viral growth. Also supporting this interaction, repressed hepcidin synthesis was attributed to hepatitis C virus (HCV) infection [107]. The UPR, whose activation has been proven in infected cells [108-110], emerges as a plausible common denominator of the aforementioned viral strategies. In fact, by exploiting the UPR pathways, viruses might simultaneously: (i) guarantee ER expansion to accommodate massive production of viral proteins, (ii) impair MHC-class I presentation [25, 26], thus helping in the immune evasion endeavor, and (iii) tune the activity of host proteins involved in iron metabolism to ensure adequate supply of this biometal.

The biological relevance of the UPR-induced modulation of iron homeostasis in the context of neurodegeneration and viral infection must be thoroughly characterized, warranting promising research directions.

\section{HH: A Candidate for Pharmacological Chaperone Therapy?}

In light of the current paradigm, the iron-dependent tuning of hepcidin is governed by the HFE/TfR1/TfR2 partnership [88]. Due to compromised cell surface expression, the C282Y variant of HFE fails to bind TfR1 or to stabilize TfR2 according to the prevailing transferrin saturation levels. Impairment of the adequate adjustment of hepcidin levels causes parenchymal iron deposition, with associated complications including cirrhosis, heart failure, diabetes, and arthropathy [4]. This loss-of-function model, however, challenges the identification of novel regulatory mechanisms mediated by the $\mathrm{C} 282 \mathrm{Y}$ mutation, as those involving its ER retention. In fact, to what extent misfolding events triggered by the $\mathrm{C} 282 \mathrm{Y}$ mutant influence the course of HFE-linked $\mathrm{HH}$ remains elusive.

The standard therapy for $\mathrm{HH}$ consists in periodic blood withdrawals - phlebotomy_aimed at depleting excessive iron stores [4]. Early initiation of treatment efficiently prevents organ failure due to iron toxicity and restores normal lifespan, although arthropathy barely improves [111]. Nevertheless, the immunological anomalies reported in $\mathrm{HH}$ patients are not solved by the blood-letting therapy [16], supporting the existence of factors beyond iron overload dictating such traits. The protective role against oxidative stress recently attributed to CRT in HFE C282Y stable transfectants, along with the negative correlation between expression of this ER chaperone and the number of clinical manifestations presented by $\mathrm{HH}$ subjects [59], conveys the rationale for considering that pharmacological chaperones might be useful in the context of HFEhemochromatosis.

The pharmacological manipulation of ER folding capacity and quality control systems has merited particular attention in recent years as promising therapeutic strategy for conformational disorders [112]. Relying on low-molecularweight compounds that stabilize native conformations and compensate intrinsic folding deficits, this approach may limit aggregation episodes and/or rescue protein function [113-116]. The endogenous bile acid taurine-conjugated 


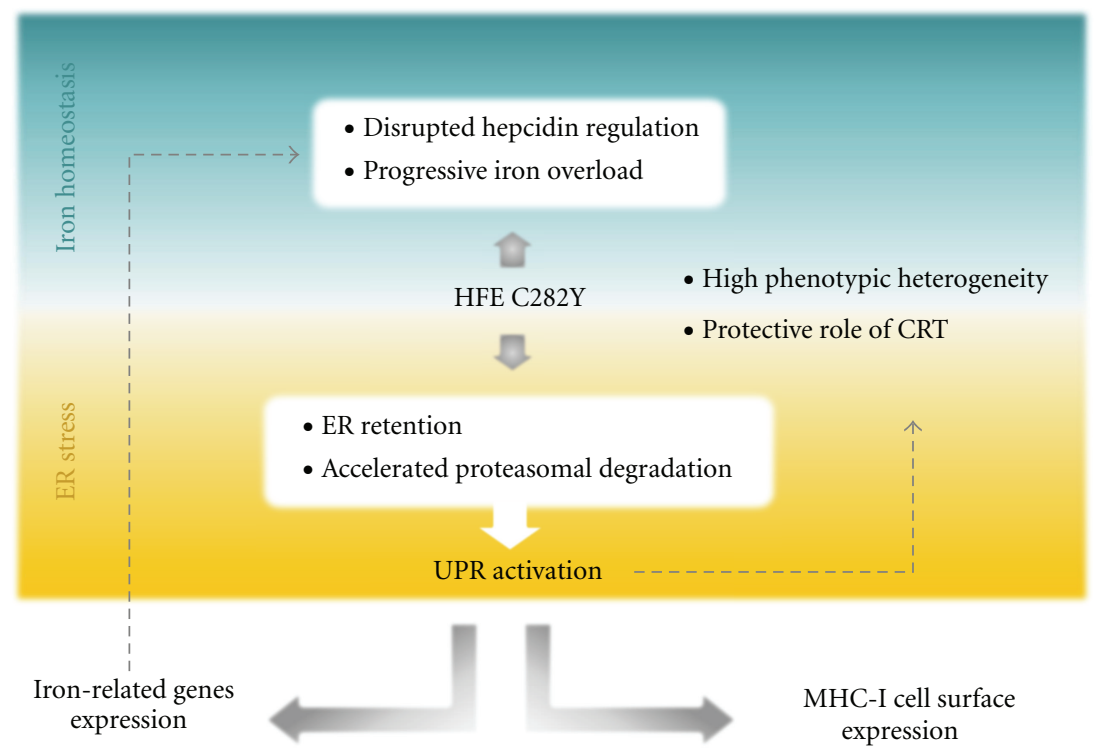

FIgURE 3: ER stressing iron homeostasis. Iron homeostasis (upper panel). Hepcidin is today thought to be the key regulator of iron homeostasis. Lower hepcidin levels have been seen associated with progression of iron overload in Hereditary Hemochromatosis (HH). $\mathrm{HH}$ is intrinsically related to the gene HFE and to a point mutation commonly known as C282Y replacing cysteine by tyrosine at position 282. A high proportion of $\mathrm{HH}$ patients are homozygous for the C282Y mutation. ER stress (lower panel). Studies by two separate groups showed in recent years that the $\mathrm{C} 282 \mathrm{Y}$ mutation in HFE provoked the activation of the Unfolded Protein Response (UPR) leading the two groups to consider $\mathrm{HH}$ a conformational disorder. In addition, some clinical phenotypic heterogeneity reported in $\mathrm{HH}$ has been related to variation in levels of mRNA expression of the endoplasmic reticulum (ER)-chaperone calreticulin (CRT). UPR activation has also been shown by separate groups to affect hepcidin gene expression. The intriguing effect of the UPR on cell surface expression of MHC class I may also contribute to the phenotypic heterogeneity of $\mathrm{HH}$ through its putative peripheral effect on numbers of circulating $\mathrm{CD} 8^{+} \mathrm{T}$ cells.

ursodeoxycholic acid (TUDCA) and the short-chain fatty acid derivate sodium 4-phenylbutyrate (4PBA) are two of such agents whose value in inhibiting the stress elicited by the HFE C282Y mutant was already established in vitro [23]. The former was shown to increase the stability of the $\mathrm{C} 282 \mathrm{Y}$ protein, whereas reduced levels of intracellular aggregates were achieved by 4 PBA supplementation. These results, underscored by the recently uncovered impact of ER stress in iron metabolism, may inspire the design of new approaches for treating HFE-HH, complementing the current clinical protocols. In this case, however, a thoroughly balanced chaperone therapy would be required in order to preserve the beneficial effects rendered by UPR activation, as those mediated by CRT against iron-generated oxidative stress [59].

\section{Concluding Remarks}

Largely propelled by its implication in varied pathological conditions, interest in understanding the interface between ER stress and other cellular signaling pathways is growing considerably. Over the last decades substantial effort to elucidate the molecular components and mechanistic details of the UPR canonical cascades was set in motion, with remarkable insights being achieved including considering a role for chaperones in therapy. Our work establishing a connection between the UPR and the expression of genes relevant for the regulation of iron metabolism reviewed in the present paper extends this networking model, highlighting further the multitasking nature of the UPR. In addition, our studies of CRT mRNA expression in genetic iron overload revealed CRT as a possible significant chaperone at the crossroads of UPR and iron homeostasis with a possible protective effect towards the iron-mediated disease due to its antioxidative properties (Figure 3 ).

Altogether, the title chosen, ER stress and iron homeostasis: a new frontier for the UPR, stresses with a few words the opening of a new promising avenue in UPR and chaperone research. It is hoped that with time facts will match the promise of words.

\section{Acknowledgments}

Authors' work was financed by Grants from Fundação para a Ciência e Tecnologia, Portugal (SFRH/BD/19682/2004 (SJO) and PTDC/BIA-BCM/66818/2006) and Innova/APBRF, USA. Authors gratefully acknowledge G. Porto for contributing with Figure 1.

\section{References}

[1] A. Helenius, T. Marquardt, and I. Braakman, "The endoplasmic reticulum as a protein-folding compartment," Trends in Cell Biology, vol. 2, no. 8, pp. 227-231, 1992.

[2] L. Ellgaard and A. Helenius, "Quality control in the endoplasmic reticulum," Nature Reviews Molecular Cell Biology, vol. 4, no. 3, pp. 181-191, 2003. 
[3] M. Schröder and R. J. Kaufman, "The mammalian unfolded protein response," Annual Review of Biochemistry, vol. 74, pp. 739-789, 2005.

[4] A. Pietrangelo, "Hereditary hemochromatosis—a new look at an old disease," New England Journal of Medicine, vol. 350, no. 23, pp. 2383-2430, 2004.

[5] J. N. Feder, A. Gnirke, W. Thomas et al., "A novel MHC class I-like gene is mutated in patients with hereditary haemochromatosis," Nature Genetics, vol. 13, no. 4, pp. 399408, 1996.

[6] J. A. Lebrón, M. J. Bennett, D. E. Vaughn et al., "Crystal structure of the hemochromatosis protein HFE and characterization of its interaction with transferrin receptor," Cell, vol. 93, no. 1, pp. 111-123, 1998.

[7] J. A. Lebrón, A. P. West Jr., and P. J. Bjorkman, "The hemochromatosis protein HFE competes with transferrin for binding to the transferrin receptor," Journal of Molecular Biology, vol. 294, no. 1, pp. 239-245, 1999.

[8] A. P. West Jr., A. M. Giannetti, A. B. Herr et al., "Mutational analysis of the transferrin receptor reveals overlapping HFE and transferrin binding sites," Journal of Molecular Biology, vol. 313, no. 2, pp. 385-397, 2001.

[9] T. Goswami and N. C. Andrews, "Hereditary hemochromatosis protein, HFE, interaction with transferrin receptor 2 suggests a molecular mechanism for mammalian iron sensing," Journal of Biological Chemistry, vol. 281, no. 39, pp. 28494-28498, 2006.

[10] A. Waheed, S. Parkkila, X. Y. Zhou et al., "Hereditary hemochromatosis: effects of $\mathrm{C} 282 \mathrm{Y}$ and $\mathrm{H} 63 \mathrm{D}$ mutations on association with $\beta 2$-microglobulin, intracellular processing, and cell surface expression of the HFE protein in COS-7 cells," Proceedings of the National Academy of Sciences of the United States of America, vol. 94, no. 23, pp. 12384-12389, 1997.

[11] E. Lyon and E. L. Frank, "Hereditary hemochromatosis since discovery of the HFE gene," Clinical Chemistry, vol. 47, no. 7, pp. 1147-1156, 2001.

[12] R. Reimao, G. Porto, and M. de Sousa, "Stability of CD4/CD8 ratios in man: new correlation between $\mathrm{CD} 4 / \mathrm{CD} 8$ profiles and iron overload in idiopathic haemochromatosis patients," Comptes Rendus de L Academie Des Sciences Serie III-Sciences de La Vie-Life Sciences, vol. 313, no. 11, pp. 481-487, 1991.

[13] G. Porto, R. Reimao, C. Goncalves, C. Vicente, B. Justica, and M. de Sousa, "Haemochromatosis as a window into the study of the immunological system: a novel correlation between CD8+ lymphocytes and iron overload," European Journal of Haematology, vol. 52, no. 5, pp. 283-290, 1994.

[14] G. Porto, C. Vicente, M. A. Teixeira et al., "Relative impact of HLA phenotype and CD4-CD8 ratios on the clinical expression of hemochromatosis," Hepatology, vol. 25, no. 2, pp. 397-402, 1997.

[15] F. A. Arosa, L. Oliveira, G. Porto et al., "Anomalies of the CD8+ T cell pool in haemochromatosis: HLA-A3linked expansions of CD8+CD28- T cells," Clinical and Experimental Immunology, vol. 107, no. 3, pp. 548-554, 1997.

[16] F. A. Arosa, A. J. da Silva, I. M. Godinho et al., "Decreased CD8-p561ck activity in peripheral blood T-lymphocytes from patients with hereditary haemochromatosis," Scandinavian Journal of Immunology, vol. 39, no. 5, pp. 426-432, 1994.

[17] M. de Sousa, R. Reimão, R. Lacerda, P. Hugo, S. H. E. Kaufmann, and G. Porto, "Iron overload in $\beta 2$-microglobulindeficient mice," Immunology Letters, vol. 39, no. 2, pp. 105111, 1994.
[18] E. Beutler, V. J. Felitti, J. A. Koziol, N. J. Ho, and T. Gelbart, "Penetrance of 845G $\rightarrow$ A (C282Y) HFE hereditary haemochromatosis mutation in the USA," Lancet, vol. 359, no. 9302, pp. 211-218, 2002.

[19] J. K. Olynyk, D. J. Cullen, S. Aquilia, E. Rossi, L. Summerville, and L. W. Powell, "A population-based study of the clinical expression of the hemochromatosis gene," New England Journal of Medicine, vol. 341, no. 10, pp. 718-724, 1999.

[20] E. Cruz, C. Whittington, S. H. Krikler et al., "A new $500 \mathrm{~kb}$ haplotype associated with high CD8+ T-lymphocyte numbers predicts a less severe expression of hereditary hemochromatosis," BMC Medical Genetics, vol. 9:97, 2008.

[21] J. N. Feder, Z. Tsuchihashi, A. Irrinki et al., "The hemochromatosis founder mutation in HLA-H disrupts $\beta 2$ - microglobulin interaction and cell surface expression," Journal of Biological Chemistry, vol. 272, no. 22, pp. 14025$14028,1997$.

[22] J. P. Pinto, P. Ramos, and M. de Sousa, "Overexpression of HFE in HepG2 cells reveals differences in intracellular distribution and co-localization of wt- and mutated forms," Blood Cells, Molecules, and Diseases, vol. 39, no. 1, pp. 75-81, 2007.

[23] S. F. de Almeida, G. Picarote, J. V. Fleming, M. CarmoFonseca, J. E. Azevedo, and M. de Sousa, "Chemical chaperones reduce endoplasmic reticulum stress and prevent mutant HFE aggregate formation," Journal of Biological Chemistry, vol. 282, no. 38, pp. 27905-27912, 2007.

[24] S. F. de Almeida, I. F. Carvalho, C. S. Cardoso et al., "HFE cross-talks with the MHC class I antigen presentation pathway," Blood, vol. 106, no. 3, pp. 971-977, 2005.

[25] S. F. de Almeida, J. V. Fleming, J. E. Azevedo, M. CarmoFonseca, and M. de Sousa, "Stimulation of an unfolded protein response impairs MHC class I expression," Journal of Immunology, vol. 178, no. 6, pp. 3612-3619, 2007.

[26] D. P. Granados, P.-L. Tanguay, M.-P. Hardy et al., "ER stress affects processing of MHC class I-associated peptides," BMC Immunology, vol. 10:10, 2009.

[27] M. W. Lawless, A. K. Mankan, M. White, M. J. O'Dwyer, and S. Norris, "Expression of hereditary hemochromatosis C282Y HFE protein in HEK293 cells activates specific endoplasmic reticulum stress responses," BMC Cell Biology, vol. 8:30, 2007.

[28] D. E. Dimcheff, S. Askovic, A. H. Baker, C. Johnson-Fowler, and J. L. Portis, "Endoplasmic reticulum stress is a determinant of retrovirus-induced spongiform neurodegeneration," Journal of Virology, vol. 77, no. 23, pp. 12617-12629, 2003.

[29] M. Calfon, H. Zeng, F. Urano et al., "IRE1 couples endoplasmic reticulum load to secretory capacity by processing the XBP-1 mRNA," Nature, vol. 415, no. 6867, pp. 92-96, 2002.

[30] S. Bernales, F. R. Papa, and P. Walter, "Intracellular signaling by the unfolded protein response," Annual Review of Cell and Developmental Biology, vol. 22, pp. 487-508, 2006.

[31] C. Xu, B. Bailly-Maitre, and J. C. Reed, "Endoplasmic reticulum stress: cell life and death decisions," Journal of Clinical Investigation, vol. 115, no. 10, pp. 2656-2664, 2005.

[32] A. Bertolotti, Y. Zhang, L. M. Hendershot, H. P. Harding, and D. Ron, "Dynamic interaction of BiP and ER stress transducers in the unfolded-protein response," Nature Cell Biology, vol. 2, no. 6, pp. 326-332, 2000.

[33] L. M. Hendershot, "The ER chaperone BiP is a master regulator of ER function," Mount Sinai Journal of Medicine, vol. 71, no. 5, pp. 289-297, 2004.

[34] J. J. Credle, J. S. Finer-Moore, F. R. Papa, R. M. Stroud, and P. Walter, "On the mechanism of sensing unfolded protein in the endoplasmic reticulum," Proceedings of the National 
Academy of Sciences of the United States of America, vol. 102, no. 52, pp. 18773-18784, 2005.

[35] J. Zhou, C. Y. Liu, S. H. Back et al., "The crystal structure of human IRE1 luminal domain reveals a conserved dimerization interface required for activation of the unfolded protein response," Proceedings of the National Academy of Sciences of the United States of America, vol. 103, no. 39, pp. 1434314348, 2006.

[36] D. Pincus, M. W. Chevalier, T. Aragón et al., "BiP binding to the ER-stress sensor Ire1 tunes the homeostatic behavior of the unfolded protein response," PLoS Biology, vol. 8, no. 7:e1000415, 2010

[37] S. F. de Almeida and M. de Sousa, "The unfolded protein response in hereditary haemochromatosis: molecular Medicine," Journal of Cellular and Molecular Medicine, vol. 12, no. 2, pp. 421-434, 2008.

[38] S. G. Gray, J. Crowe, and M. W. Lawless, "Hemochromatosis: as a conformational disorder," International Journal of Biochemistry and Cell Biology, vol. 41, no. 11, pp. 2094-2097, 2009.

[39] M. Chloupková, A.-S. Zhang, and C. A. Enns, "Stoichiometries of transferrin receptors 1 and 2 in human liver," Blood Cells, Molecules, and Diseases, vol. 44, no. 1, pp. 28-33, 2009.

[40] A. Bondi, P. Valentino, F. Daraio et al., "Hepatic expression of hemochromatosis genes in two mouse strains after phlebotomy and iron overload," Haematologica, vol. 90, no. 9, pp. 1161-1167, 2005.

[41] S. Ludwiczek, I. Theurl, E. Artner-Dworzak, M. Chorney, and G. Weiss, "Duodenal HFE expression and hepcidin levels determine body iron homeostasis: modulation by genetic diversity and dietary iron availability," Journal of Molecular Medicine, vol. 82, no. 6, pp. 373-382, 2004.

[42] I. Theurl, S. Ludwiczek, P. Eller et al., "Pathways for the regulation of body iron homeostasis in response to experimental iron overload," Journal of Hepatology, vol. 43, no. 4, pp. 711-719, 2005.

[43] S. Y. Lee and J. R. Connor, "Regulation of Hfe by stress factors in BV-2 cells," Neurobiology of Aging, vol. 26, no. 6, pp. 803812, 2005.

[44] A. M. Reimold, N. N. Iwakoshi, J. Manis et al., "Plasma cell differentiation requires the transcription factor XBP-1," Nature, vol. 412, no. 6844, pp. 300-307, 2001.

[45] W. Zhang, D. Feng, Y. Li, K. Iida, B. McGrath, and D. R. Cavener, "PERK EIF2AK3 control of pancreatic $\beta$ cell differentiation and proliferation is required for postnatal glucose homeostasis," Cell Metabolism, vol. 4, no. 6, pp. 491497, 2006.

[46] K. Zhang, X. Shen, J. Wu et al., "Endoplasmic reticulum stress activates cleavage of CREBH to induce a systemic inflammatory response," Cell, vol. 124, no. 3, pp. 587-599, 2006.

[47] Z. Ye and J. R. Connor, "Identification of iron responsive genes by screening cDNA libraries from suppression subtractive hybridization with antisense probes from three iron conditions," Nucleic Acids Research, vol. 28, no. 8, pp. 18021807, 2000.

[48] K.-R. You, M.-J. Liu, X.-J. Han, Z.-W. Lee, and D.-G. Kim, "Transcriptional regulation of the human transferrin gene by GADD153 in hepatoma cells," Hepatology, vol. 38, no. 3, pp. 745-755, 2003.

[49] J. Petrak, D. Myslivcova, P. Man et al., "Proteomic analysis of hepatic iron overload in mice suggests dysregulation of urea cycle, impairment of fatty acid oxidation, and changes in the methylation cycle," American Journal of Physiology - Gastrointestinal and Liver Physiology, vol. 292, no. 6, pp. G1490-G1498, 2007.

[50] S. J. Oliveira, J. P. Pinto, G. Picarote et al., "ER stressinducible factor $\mathrm{CHOP}$ affects the expression of hepcidin by modulating C/EBPalpha activity," PLoS ONE, vol. 4, no. 8:e6618, 2009.

[51] C. Vecchi, G. Montosi, K. Zhang et al., "ER stress controls iron metabolism through induction of hepcidin," Science, vol. 325, no. 5942, pp. 877-880, 2009.

[52] E. M. Conway, L. Liu, B. Nowakowski, M. Steiner-Mosonyi, S. P. Ribeiro, and M. Michalak, "Heat shock-sensitive expression of calreticulin. In vitro and in vivo up-regulation," Journal of Biological Chemistry, vol. 270, no. 28, pp. 1701117016, 1995.

[53] T. Q. Nguyen, J. D. Capra, and R. D. Sontheimer, "Calreticulin is transcriptionally upregulated by heat shock, calcium and heavy metals," Molecular Immunology, vol. 33, no. 4-5, pp. 379-386, 1996.

[54] M. Szewczenko-Pawlikowski, E. Dziak, M. J. McLaren, M. Michalak, and M. Opas, "Heat shock-regulated expression of calreticulin in retinal pigment epithelium," Molecular and Cellular Biochemistry, vol. 177, no. 1-2, pp. 145-152, 1997.

[55] A. E. Crib, M. Peyrou, S. Muruganandan, and L. Schneider, "The endoplasmic reticulum in xenobiotic toxicity," Drug Metabolism Reviews, vol. 37, no. 3, pp. 405-442, 2005.

[56] H. Liu, R. C. Bowes III, B. Van de Water, C. Sillence, J. F. Nagelkerke, and J. L. Stevens, "Endoplasmic reticulum chaperones GRP78 and calreticulin prevent oxidative stress, $\mathrm{Ca} 2+$ disturbances, and cell death in renal epithelial cells," Journal of Biological Chemistry, vol. 272, no. 35, pp. 2175121759, 1997.

[57] S. Oyadomari, K. Takeda, M. Takiguchi et al., "Nitric oxideinduced apoptosis in pancreatic $\beta$ cells is mediated by the endoplasmic reticulum stress pathway," Proceedings of the National Academy of Sciences of the United States of America, vol. 98, no. 19, pp. 10845-10850, 2001.

[58] F. F. Xu, X. H. Liu, and M. Zhu, "Calreticulin upregulation induced by hypoxic preconditioning relieves oxidative stress injury in rat cardiomyocytes," Sheng Li Xue Bao, vol. 60, no. 1, pp. 29-37, 2008.

[59] J. P. Pinto, P. Ramos, S. F. de Almeida et al., "Protective role of calreticulin in HFE hemochromatosis," Free Radical Biology and Medicine, vol. 44, no. 1, pp. 99-108, 2008.

[60] M. T. Núñez, A. Osorio, V. Tapia, A. Vergara, and C. V. Mura, "Iron-induced oxidative stress up-regulates calreticulin levels in intestinal epithelial (Caco-2) cells," Journal of Cellular Biochemistry, vol. 82, no. 4, pp. 660-665, 2001.

[61] M. E. Conrad, J. N. Umbreit, E. G. Moore, and K. P. Harper, "Iron absorption via the mucin-integrin mobilferrin pathway," Transactions of the Association of American Physicians, vol. 105, pp. 133-148, 1992.

[62] M. E. Conrad, J. N. Umbreit, and E. G. Moore, "Rat duodenal iron-binding protein mobilferrin is a homologue of calreticulin," Gastroenterology, vol. 104, no. 6, pp. 17001704, 1993.

[63] M. Dizdaroglu, "Oxidative damage to DNA in mammalian chromatin," Mutation Research, vol. 275, no. 3-6, pp. 331342, 1992.

[64] B. Tudek, A. Winczura, J. Janik, A. Siomek, M. Foksinski, and R. Oliński, "Involvement of oxidatively damaged DNA and repair in cancer development and aging," American Journal of Translational Research, vol. 2, no. 3, pp. 254-284, 2010. 
[65] A. A. Alkhateeb and J. R. Connor, "Nuclear ferritin: a new role for ferritin in cell biology," Biochimica et Biophysica Acta, vol. 1800, no. 8, pp. 793-797, 2010.

[66] A. G. Smith, P. Carthew, J. E. Francis, R. E. Edwards, and D. Dinsdale, "Characterization and accumulation of ferritin in hepatocyte nuclei of mice with iron overload," Hepatology, vol. 12, no. 6, pp. 1399-1405, 1990.

[67] C. X. Cai, D. E. Birk, and T. F. Linsenmayer, "Nuclear ferritin protects DNA from UV damage in corneal epithelial cells," Molecular Biology of the Cell, vol. 9, no. 5, pp. 1037-1051, 1998.

[68] C. Cai, A. Ching, C. Lagace, and T. Linsenmayer, "Nuclear ferritin-mediated protection of corneal epithelial cells from oxidative damage to DNA," Developmental Dynamics, vol. 237, no. 10, pp. 2676-2683, 2008.

[69] J. P. Rabek, W. H. Boylston III, and J. Papaconstantinou, "Carbonylation of ER chaperone proteins in aged mouse liver," Biochemical and Biophysical Research Communications, vol. 305, no. 3, pp. 566-572, 2003.

[70] L. I. Gold, P. Eggleton, M. T. Sweetwyne et al., "Calreticulin: non-endoplasmic reticulum functions in physiology and disease," FASEB Journal, vol. 24, no. 3, pp. 665-683, 2010.

[71] N. Casares, M. O. Pequignot, A. Tesniere et al., "Caspasedependent immunogenicity of doxorubicin-induced tumor cell death," Journal of Experimental Medicine, vol. 202, no. 12, pp. 1691-1701, 2005.

[72] M. Obeid, T. Panaretakis, A. Tesniere et al., "Leveraging the immune system during chemotherapy: moving calreticulin to the cell surface converts apoptotic death from "silent" to immunogenic," Cancer Research, vol. 67, no. 17, pp. 79417944, 2007.

[73] R. Tufi, T. Panaretakis, K. Bianchi et al., "Reduction of endoplasmic reticulum $\mathrm{Ca} 2+$ levels favors plasma membrane surface exposure of calreticulin," Cell Death and Differentiation, vol. 15, no. 2, pp. 274-282, 2008.

[74] T. Panaretakis, O. Kepp, U. Brockmeier et al., "Mechanisms of pre-apoptotic calreticulin exposure in immunogenic cell death," EMBO Journal, vol. 28, no. 5, pp. 578-590, 2009.

[75] E. M. Cardoso, M. G. Macedo, P. Rohrlich et al., "Increased hepatic iron in mice lacking classical MHC class I molecules," Blood, vol. 100, no. 12, pp. 4239-4241, 2002.

[76] D. R. Peaper and P. Cresswell, "Regulation of MHC class I assembly and peptide binding," Annual Review of Cell and Developmental Biology, vol. 24, pp. 343-368, 2008.

[77] P. M. Rudd, T. Elliott, P. Cresswell, I. A. Wilson, and R. A. Dwek, "Glycosylation and the immune system," Science, vol. 291, no. 5512, pp. 2370-2376, 2001.

[78] T. Elliott and A. Williams, "The optimization of peptide cargo bound to MHC class I molecules by the peptideloading complex," Immunological Reviews, vol. 207, pp. 8999, 2005.

[79] P. A. Wearsch, C. A. Jakob, A. Vallin, R. A. Dwek, P. M. Rudd, and P. Cresswell, "Major histocompatibility complex class I molecules expressed with monoglucosylated N-linked glycans bind calreticulin independently of their assembly status," Journal of Biological Chemistry, vol. 279, no. 24, pp. 25112-25121, 2004.

[80] B. Gao, R. Adhikari, M. Howarth et al., "Assembly and antigen-presenting function of MHC class I molecules in cells lacking the ER chaperone calreticulin," Immunity, vol. 16, no. 1, pp. 99-109, 2002.

[81] B. S. Ireland, U. Brockmeier, C. M. Howe, T. Elliott, and D. B. Williams, "Lectin-deficient calreticulin retains full functionality as a chaperone for class I histocompatibility molecules," Molecular Biology of the Cell, vol. 19, no. 6, pp. 2413-2423, 2008.

[82] C. H. Park, E. V. Valore, A. J. Waring, and T. Ganz, "Hepcidin, a urinary antimicrobial peptide synthesized in the liver," Journal of Biological Chemistry, vol. 276, no. 11, pp. 78067810, 2001.

[83] E. Nemeth, M. S. Tuttle, J. Powelson et al., "Hepcidin regulates cellular iron efflux by binding to ferroportin and inducing its internalization," Science, vol. 306, no. 5704, pp. 2090-2093, 2004.

[84] C. Pigeon, G. Ilyin, B. Courselaud et al., "A new mouse liver-specific gene, encoding a protein homologous to human antimicrobial peptide hepcidin, is overexpressed during iron overload," Journal of Biological Chemistry, vol. 276, no. 11, pp. 7811-7819, 2001.

[85] G. Nicolas, C. Chauvet, L. Viatte et al., "The gene encoding the iron regulatory peptide hepcidin is regulated by anemia, hypoxia, and inflammation," Journal of Clinical Investigation, vol. 110, no. 7, pp. 1037-1044, 2002.

[86] R.-H. Wang, C. Li, X. Xu et al., "A role of SMAD4 in iron metabolism through the positive regulation of hepcidin expression," Cell Metabolism, vol. 2, no. 6, pp. 399-409, 2005.

[87] J. L. Babitt, F. W. Huang, D. M. Wrighting et al., "Bone morphogenetic protein signaling by hemojuvelin regulates hepcidin expression," Nature Genetics, vol. 38, no. 5, pp. 531539, 2006.

[88] J. Gao, J. Chen, M. Kramer, H. Tsukamoto, A.-S. Zhang, and C. A. Enns, "Interaction of the hereditary hemochromatosis protein HFE with transferrin receptor 2 is required for transferrin-induced hepcidin expression," Cell Metabolism, vol. 9, no. 3, pp. 217-227, 2009.

[89] D. M. Wrighting and N. C. Andrews, "Interleukin-6 induces hepcidin expression through STAT3,” Blood, vol. 108, no. 9, pp. 3204-3209, 2006.

[90] C. Peyssonnaux, A. S. Zinkernagel, R. A. Schuepbach et al., "Regulation of iron homeostasis by the hypoxia-inducible transcription factors (HIFs)," Journal of Clinical Investigation, vol. 117, no. 7, pp. 1926-1932, 2007.

[91] J. P. Pinto, S. Ribeiro, H. Pontes et al., "Erythropoietin mediates hepcidin expression in hepatocytes through EPOR signaling and regulation of C/EBP $\alpha$, Blood, vol. 111, no. 12, pp. 5727-5733, 2008.

[92] K. L. Lipson, S. G. Fonseca, S. Ishigaki et al., "Regulation of insulin biosynthesis in pancreatic beta cells by an endoplasmic reticulum-resident protein kinase IRE1," Cell Metabolism, vol. 4, no. 3, pp. 245-254, 2006.

[93] U. Özcan, Q. Cao, E. Yilmaz et al., "Endoplasmic reticulum stress links obesity, insulin action, and type 2 diabetes," Science, vol. 306, no. 5695, pp. 457-461, 2004.

[94] J. C. Gonzales, C. L. Gentile, K. T. Pfaffenbach, Y. Wei, D. Wang, and M. J. Pagliassotti, "Chemical induction of the unfolded protein response in the liver increases glucose production and is activated during insulin-induced hypoglycaemia in rats," Diabetologia, vol. 51, no. 10, pp. 1920-1929, 2008.

[95] K. Zhang and R. J. Kaufman, "From endoplasmic-reticulum stress to the inflammatory response," Nature, vol. 454, no. 7203, pp. 455-462, 2008.

[96] F. Martinon, X. Chen, A. -H. Lee, and L. H. Glimcher, "TLR activation of the transcription factor XBP1 regulates innate immune responses in macrophages," Nature Immunology, vol. 11, no. 5, pp. 411-418, 2010. 
[97] S. Oyadomari, E. Araki, and M. Mori, "Endoplasmic reticulum stress-mediated apoptosis in pancreatic $\beta$-cells," Apoptosis, vol. 7, no. 4, pp. 335-345, 2002.

[98] L. Ozcan, A. S. Ergin, A. Lu et al., "Endoplasmic reticulum stress plays a central role in development of leptin resistance," Cell Metabolism, vol. 9, no. 1, pp. 35-51, 2009.

[99] S. Matus, F. Lisbona, M. Torres, C. León, P. Thielen, and C. Hetz, "The stress rheostat: an interplay between the unfolded protein response (UPR) and autophagy in neurodegeneration," Current Molecular Medicine, vol. 8, no. 3, pp. 157-172, 2008.

[100] L. Zecca, M. B. H. Youdim, P. Riederer, J. R. Connor, and R. R. Crichton, "Iron, brain ageing and neurodegenerative disorders," Nature Reviews Neuroscience, vol. 5, no. 11, pp. 863-873, 2004.

[101] S. Altamura and M. U. Muckenthaler, "Iron toxicity in diseases of aging: Alzheimer's disease, Parkinson's disease and atherosclerosis," Journal of Alzheimer's Disease, vol. 16, no. 4, pp. 879-895, 2009.

[102] E. W. Hewitt, "The MHC class I antigen presentation pathway: strategies for viral immune evasion," Immunology, vol. 110, no. 2, pp. 163-169, 2003.

[103] J. S. L. Parker and C. R. Parrish, "Cellular uptake and infection by canine parvovirus involves rapid dynaminregulated clathrin-mediated endocytosis, followed by slower intracellular trafficking," Journal of Virology, vol. 74, no. 4, pp. 1919-1930, 2000.

[104] J. S. L. Parker, W. J. Murphy, D. Wang, S. J. O’Brien, and C. R. Parrish, "Canine and feline parvoviruses can use human or feline transferrin receptors to bind, enter, and infect cells," Journal of Virology, vol. 75, no. 8, pp. 3896-3902, 2001.

[105] S. V. Ben-Arieh, B. Zimerman, N. I. Smorodinsky et al., "Human cytomegalovirus protein US2 interferes with the expression of human HFE, a nonclassical class I major histocompatibility complex molecule that regulates iron homeostasis," Journal of Virology, vol. 75, no. 21, pp. 1055710562, 2001.

[106] H. Drakesmith, N. Chen, H. Ledermann, G. Screaton, A. Townsend, and X.-N. Xu, "HIV-1 Nef down-regulates the hemochromatosis protein HFE, manipulating cellular iron homeostasis," Proceedings of the National Academy of Sciences of the United States of America, vol. 102, no. 31, pp. 1101711022, 2005.

[107] S. Nishina, K. Hino, M. Korenaga et al., "Hepatitis C virusinduced reactive oxygen species raise hepatic iron level in mice by reducing hepcidin transcription," Gastroenterology, vol. 134, no. 1, pp. 226-238, 2008.

[108] K. D. Tardif, K. Mori, R. J. Kaufman, and A. Siddiqui, "Hepatitis C virus suppresses the IRE1-XBP1 pathway of the unfolded protein response," Journal of Biological Chemistry, vol. 279, no. 17, pp. 17158-17164, 2004.

[109] J. A. Isler, A. H. Skalet, and J. C. Alwine, "Human cytomegalovirus infection activates and regulates the unfolded protein response," Journal of Virology, vol. 79, no. 11, pp. 6890-6899, 2005.

[110] B. Li, B. Gao, L. Ye et al., "Hepatitis B virus X protein ( $\mathrm{HBx})$ activates ATF6 and IRE1-XBP1 pathways of unfolded protein response," Virus Research, vol. 124, no. 1-2, pp. 44-49, 2007.

[111] A. S. Tavill, "Diagnosis and management of hemochromatosis," Hepatology, vol. 33, no. 5, pp. 1321-1328, 2001.

[112] V. Bernier, M. Lagacé, D. G. Bichet, and M. Bouvier, "Pharmacological chaperones: potential treatment for conformational diseases," Trends in Endocrinology and Metabolism, vol. 15, no. 5, pp. 222-228, 2004.
[113] K. Kubota, Y. Niinuma, M. Kaneko et al., "Suppressive effects of 4-phenylbutyrate on the aggregation of Pael receptors and endoplasmic reticulum stress," Journal of Neurochemistry, vol. 97, no. 5, pp. 1259-1268, 2006.

[114] J. A.J. Burrows, L. K. Willis, and D. H. Perlmutter, "Chemical chaperones mediate increased secretion of mutant $\alpha 1$ antitrypsin $(\alpha 1-\mathrm{AT}) \mathrm{Z}$ : a potential pharmacological strategy for prevention of liver injury and emphysema in $\alpha 1$-AT deficiency," Proceedings of the National Academy of Sciences of the United States of America, vol. 97, no. 4, pp. 1796-1801, 2000.

[115] J.-P. Morello, A. Salahpour, A. Laperrière et al., "Pharmacological chaperones rescue cell-surface expression and function of misfolded V2 vasopressin receptor mutants," Journal of Clinical Investigation, vol. 105, no. 7, pp. 887-895, 2000.

[116] Y. Wang, T. W. Loo, M. C. Bartlett, and D. M. Clarke, "Additive effect of multiple pharmacological chaperones on maturation of CFTR processing mutants," Biochemical Journal, vol. 406, no. 2, pp. 257-263, 2007. 

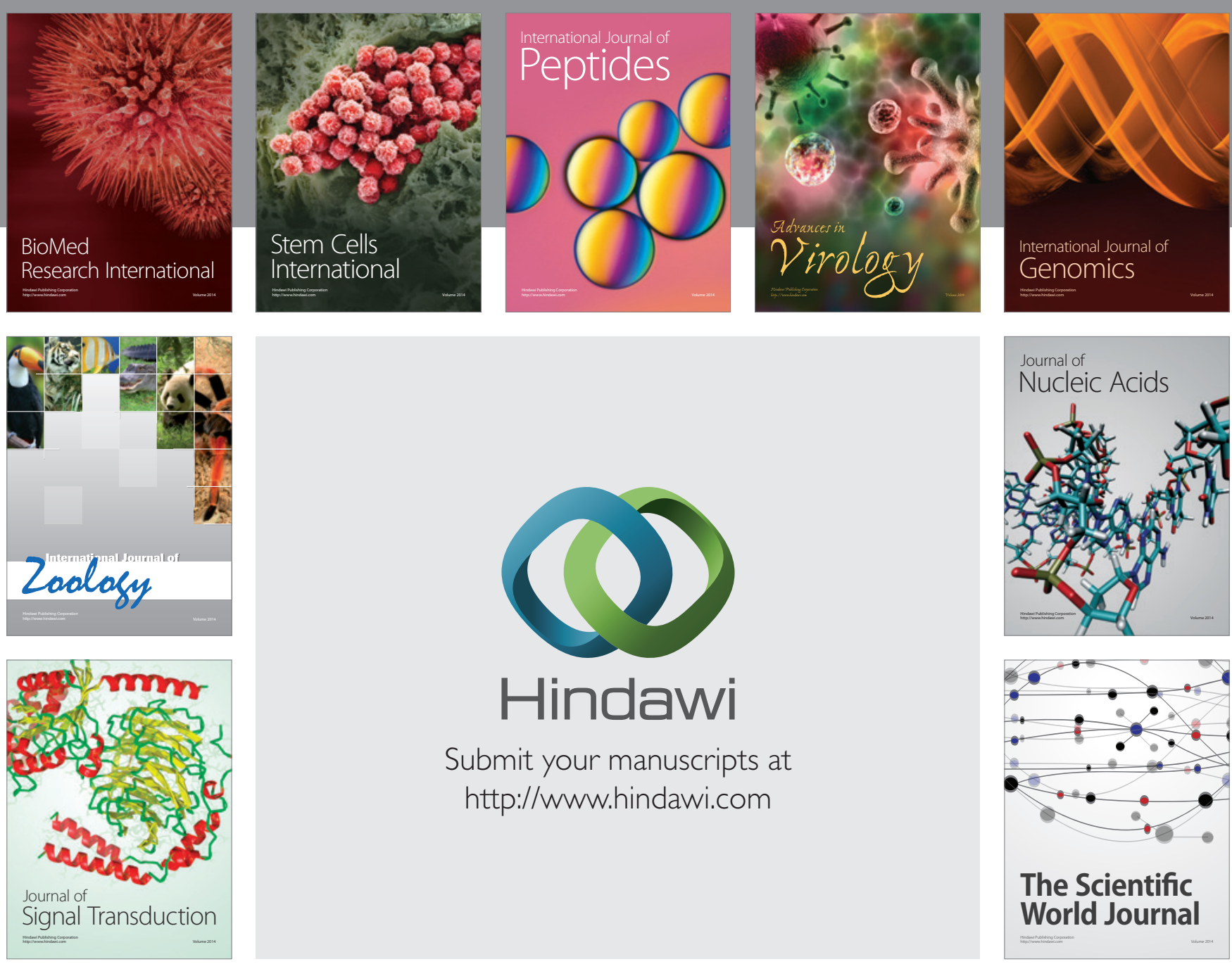

Submit your manuscripts at

http://www.hindawi.com
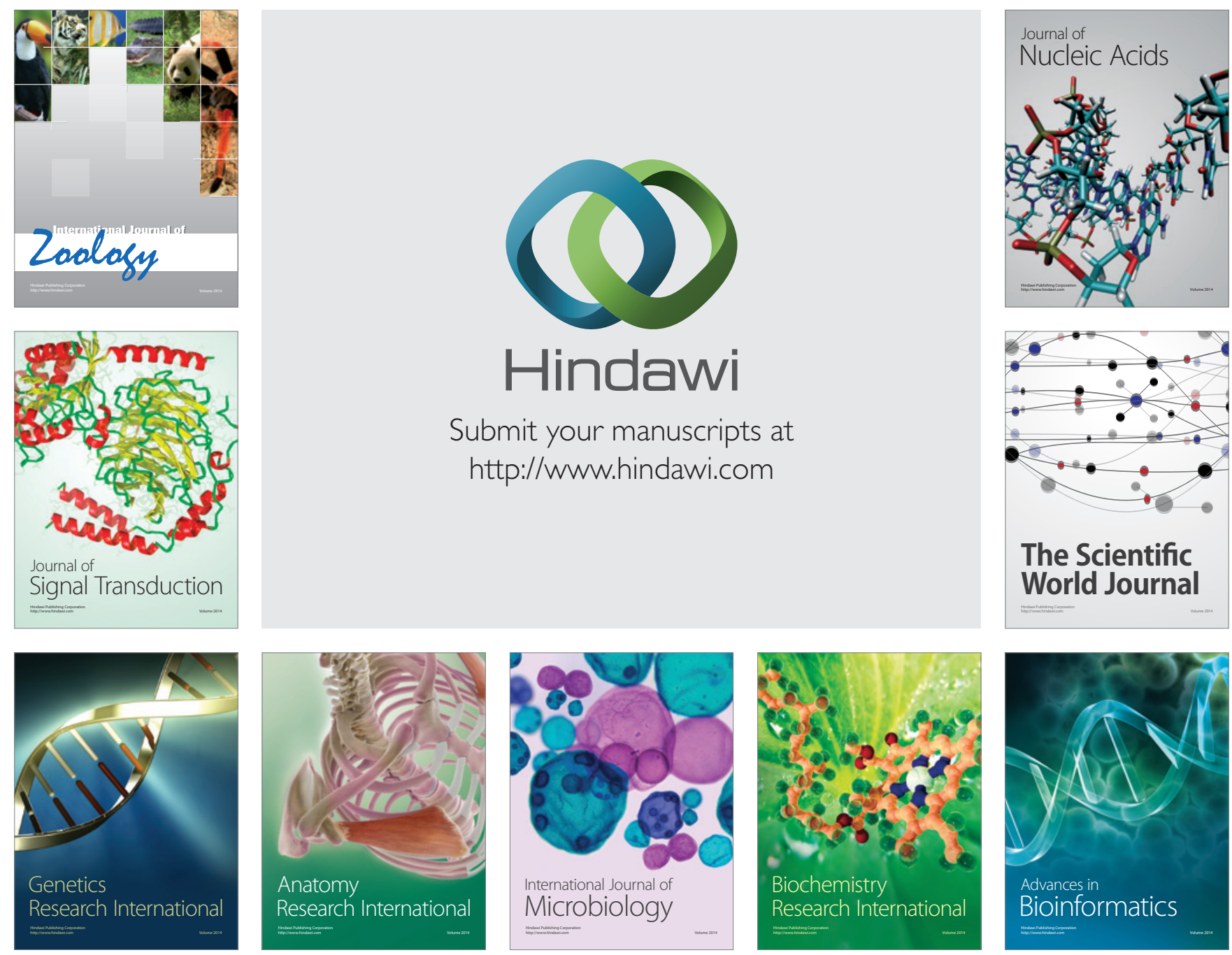

The Scientific World Journal
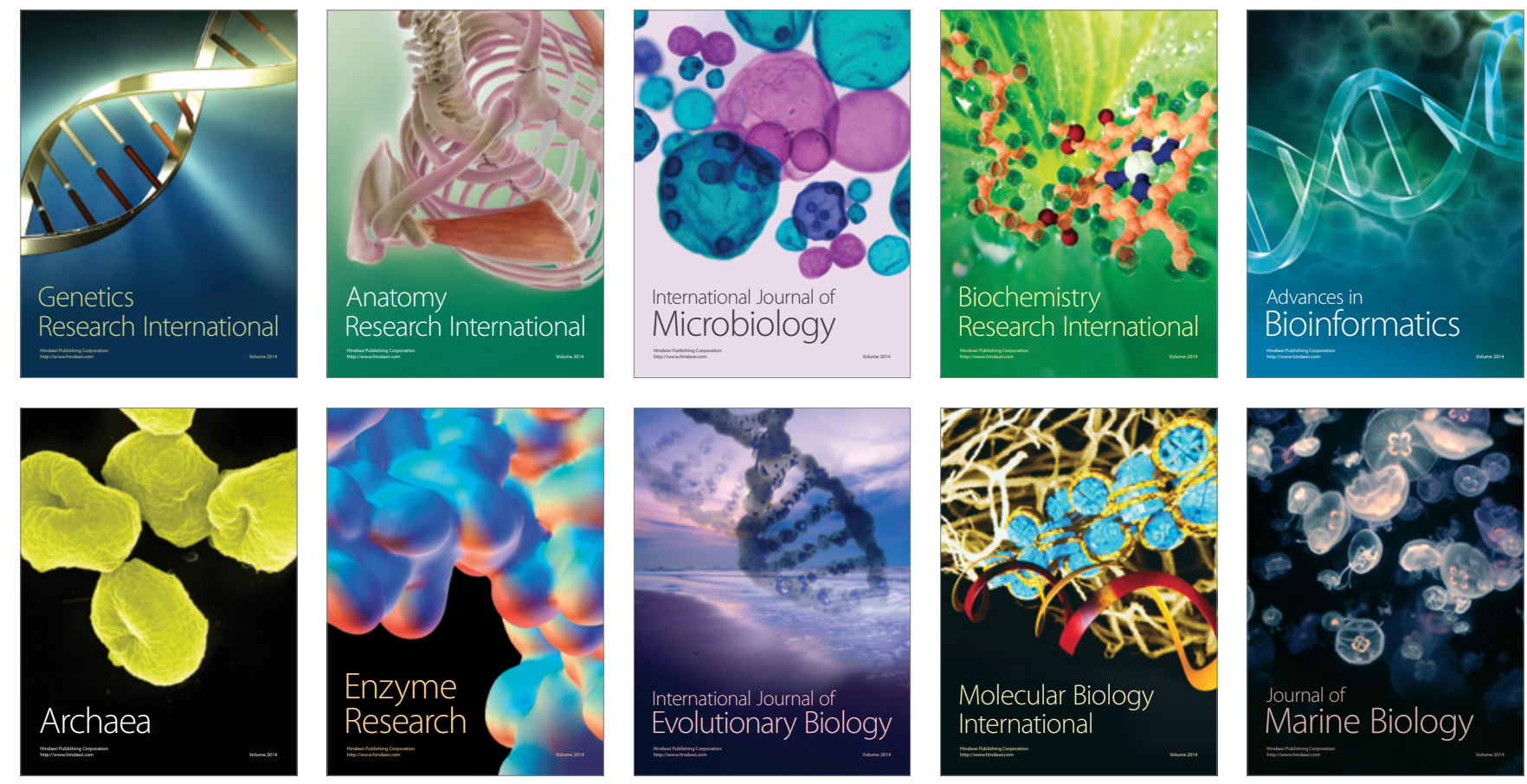